\title{
Metabolic and Bariatric Surgery Chapter of the Association of Polish Surgeons. Bariatric and metabolic surgery care standards
}

\author{
Jacek Szeliga ${ }^{1}$, Mariusz Wyleżoł², Piotr Major ${ }^{3}$, Andrzej Budzyński ${ }^{4}$, Artur Binda ${ }^{5}$, Monika Proczko-Stepaniak \\ Iwona Boniecka ${ }^{7}$, Maciej Matłok ${ }^{8}$, Marzena Sekuła ${ }^{9}$, Łukasz Kaska ${ }^{6}$, Piotr Myśliwiec ${ }^{10}$, Tomasz Szewczyk ${ }^{11}$, \\ Marcin Możański ${ }^{12}$, Grzegorz Kowalski ${ }^{13}$, Wiesław Pesta ${ }^{14}$, Wojciech Lisik $^{15}$, Maciej Michalik ${ }^{16}$, \\ Tomasz Lewandowski ${ }^{17}$, Krzysztof Paśnik ${ }^{1}$ \\ ${ }^{1}$ Department of General, Gastroenterological, and Oncological Surgery, Collegium Medicum in Bydgoszcz, \\ Nicolaus Copernicus University in Torun, Poland \\ ${ }^{2}$ Department of General, Vascular, and Oncological Surgery, Medical University of Warsaw, Poland \\ ${ }^{3} 2^{\text {nd }}$ Department of General Surgery, Jagiellonian University Medical College, Krakow, Poland \\ ${ }^{4}$ Department of General and Oncological Surgery, Ludwik Rydygier Memorial Hospital, Krakow, Poland \\ ${ }^{5}$ Department of General, Oncological, and Digestive Tract Surgery, Medical Centre of Postgraduate Education, Orlowski Hospital, \\ Warsaw, Poland \\ ${ }^{6}$ Department of General, Endocrine and Transplant Surgery, Gdansk Medical University, Poland \\ ${ }^{7}$ Department of Clinical Dietetics, Medical University of Warsaw, Poland \\ ${ }^{8}$ Department of Endoscopic and Metabolic Surgery, Specialised Hospital Pro-Familia, Rzeszow, Poland \\ ${ }^{9}$ SWPS University of Social Sciences and Humanities, Warsaw, Poland \\ ${ }^{10} 1^{\text {st }}$ Clinical Department of General and Endocrine Surgery, Medical University of Bialystok, Poland \\ ${ }^{11}$ Department of General Surgery, RCZ, Lubin, Poland \\ ${ }^{12}$ Clinical Department of Anaesthesiology and Intensive Care, Military Medical Institute, Warsaw, Poland \\ ${ }^{13}$ Department of General and Endocrinological Surgery, Medical University of Silesia, Katowice, Poland \\ ${ }^{14}$ Department of General, Metabolic, and Bariatric Surgery, University of Warmia-Mazury, Olsztyn, Poland \\ ${ }^{15}$ Department of General Surgery and Transplantology, Medical University of Warsaw, Poland \\ ${ }^{16} \mathrm{Clinic}$ of General, Minimally Invasive, and Elderly Surgery, University of Warmia and Mazury, Olsztyn, Poland \\ ${ }^{17}$ Department of General Surgery with Subunit of Vascular Surgery, $1^{\text {st }}$ Military Clinical Hospital with Outpatient Clinic in Lublin, \\ Affiliate in Ełk, Poland
}

Videosurgery Mininv DOI: https://doi.org/10.5114/wiitm.2020.97935

\begin{abstract}
The Metabolic and Bariatric Surgery Chapter of the Association of Polish Surgeons (Polish acronym: SCMiB TCHP) is a Polish specialist scientific society representing bariatric surgeons as well as specialists from other disciplines and professions cooperating with them during the provision of services in the field of bariatric and metabolic surgery, as well as the entire care process before and after surgery. The following standards constitute the minimum requirements set by the SCMiB TCHP for good practice of the basic process of bariatric care throughout its entire period, which ensure satisfactory safety and effectiveness of the obesity treatment and its metabolic complications.
\end{abstract}

Key words: bariatric surgery, obesity, metabolic surgery.

\section{Address for correspondence}

Jacek Szeliga MD, PhD, Prof. UMK, Department of General, Gastroenterological, and Oncological Surgery, Collegium Medicum, Nicolaus Copernicus University, Bydgoszcz, Poland, phone: +48 509207 072, e-mail: jacky2@wp.pl 
Jacek Szeliga, Mariusz Wyleżoł, Piotr Major, Andrzej Budzyński, Artur Binda, Monika Proczko-Stepaniak, Iwona Boniecka, Maciej Matłok, Marzena Sekuła, Łukasz Kaska, Piotr Myśliwiec, Tomasz Szewczyk, Marcin Możański, Grzegorz Kowalski, Wiesław Pesta, Wojciech Lisik, Maciej Michalik, Tomasz Lewandowski, Krzysztof Paśnik

\section{Introduction}

In developed and developing countries alike, the incidence of obesity is increasing rapidly, reaching epidemic proportions. Over the past 30 years, the number of obese people in the world has increased threefold and affects most countries of the world. Currently, the epidemiological problem of obesity is independent of specific socioeconomic groups. As early as in 1998, the World Health Organisation (WHO) recognised obesity and its complications as the most serious chronic health problem in the modern world. Based on data from 2016, it has been estimated that worldwide about $39 \%$ of the population over age 18 years is affected by the problem of excessive weight, of which nearly 600 million persons are obese.

Bariatric surgery has been scientifically accepted as the only clinically and economically effective way to currently treat obesity. It has ceased to be a treatment only for excessive body weight; much more important than this are the metabolic effects of the procedure leading to the resolution of complications of the underlying disease.

The Metabolic and Bariatric Surgery Chapter of the Association of Polish Surgeons (Polish acronym: SCMiB TCHP) is a Polish specialist scientific society representing bariatric surgeons as well as specialists from other disciplines and professions cooperating with them during the provision of services in the field of bariatric and metabolic surgery, as well as the entire care process before and after surgery.

The Section of Metabolic and Bariatric Surgery is accredited by the International Federation of Obesity Surgery (IFSO).

The following standards constitute the minimum requirements set by the SCMiB TCHP for good practice of the basic process of bariatric care throughout its entire period, which ensure satisfactory safety and effectiveness of the obesity treatment and its metabolic complications. In situations requiring separate, individualised management, it is recommended that they be conducted based on the "Polish recommendations in the field of bariatric and metabolic surgery" [1].

The resulting standards are addressed to the following:

a) National Health Foundation representatives,

b) clinicians and leaders in medical services,

c) administrators of medical units,

d) independent private sector entities.
These standards will be periodically updated due to the constant advances in medical science. However, they constitute the current consensus gathered from among the SCMiB TCHP members, and they represent the official position that the Association of Polish Surgeons will use if asked for an opinion in a given situation.

The structure of standards is based on the individual stages of the treatment of obesity and its complications:

a) preoperative period (covering the period from qualification up to surgery),

b) perioperative period (from surgery up to 30 days after surgery),

c) postoperative period (from day 31 up to the patient's death).

Expected benefits:

a) for the patient:

- placing the patient at the centre of attention of the medical staff who care for them,

- facilitating the best decision regarding the management of the obese patient's care,

- unifying the medical protocol used by medical staff, regardless of where healthcare services are provided;

b) for medical staff:

- ensuring proper and uniform organisation of care for obese patients, in the most effective manner, using the potential of the staff, while guaranteeing patient safety,

- ensuring a sense of security for patients as well as staff who follow the principles,

- contribute to reducing the number of medical interventions not arising from medical indications,

- establishing quality indicators for bariatric care and the capability of monitoring them.

\section{Care standards in the preoperative period}

Care standards in the preoperative period (from qualification for bariatric treatment to admission to the centre for surgery)

1. Qualification for the treatment of obesity by surgical methods

The patient eligibility criteria for operative treatment of morbid obesity are based on the assessment of the body mass index (BMI), defined as the patient's weight expressed in kilograms divided by the square of height expressed in metres. 
The indication for the surgical treatment of morbid obesity are the patient's highest documented body mass index (BMI) values:

a) BMI equal to or greater than $40 \mathrm{~kg} / \mathrm{m}^{2}$,

b) BMI in the range of $35-40 \mathrm{~kg} / \mathrm{m}^{2}$ in patients in whom surgically induced weight reduction can bring potential improvement in diseases caused by obesity alone,

c) BMI higher than $30 \mathrm{~kg} / \mathrm{m}^{2}$ in patients with diabetes type 2 who do not respond to drug treatment.

2. Qualification for bariatric treatment is carried out by a surgeon performing bariatric (metabolic) surgeries, and who is a member of the bariatric team.

3. Preparation for surgery is a process supervised by a surgeon, which has to include the following:

a) consultations of a dietitian with experience in caring for bariatric patients - 2 (initial and final care), including an assessment of the effect of applying initial dietary recommendations and education on nutrition in the peri- and postoperative period,

b) a minimum $5 \%$ reduction in body weight/fat,

c) consultation - a visit to a psychologist with experience in caring for bariatric patients including an assessment of potential contraindications for bariatric treatment (At the time of publication of these standards, the service was not financed by the National Health Fund),

d) consultation - a visit to an anaesthesiologist,

e) laboratory test panel necessary to perform the elective surgery under general anaesthesia in a given centre,

f) screening for TSH, cortisol, fasting glucose,

g) glycated haemoglobin $\left(\mathrm{HbA}_{1 \mathrm{c}}\right)$ determination in patients with diabetes mellitus type 2 ,

h) determination of parameters related to the assessment of nutritional status, on the basis of which it is later possible to evaluate the results of treatment and the effects of applying dietary recommendations,

i) imaging diagnostics: EGD, abdominal ultrasound,

j) ECG,

k) obstructive sleep apnoea risk assessment (OSA) according to the STOP-Bang questionnaire, and if the result is $\geq 6$, the referral for sleep disorder examination is necessary to qualify for CPAP treatment,

l) obtaining a patient's declaration of abstaining from smoking for a minimum of 6 weeks before the planned surgery, m) obtaining patient's informed consent for surgery, confirmed by signing the consent form recommended by the Association of Polish Surgeons.

\section{Care standards during the perioperative period}

Care standards during the perioperative period (from admission to hospital up to 30 days after surgery)

1. The surgical bariatric team has to include at least one surgeon - a specialist with experience in bariatric surgery, who meets the requirements of the SCMiB certification.

2. Bariatric facility equipment has to meet the following requirements:

The following must be available at the place of providing the service: 1 ) equipment adapted to patients weighing up to $250 \mathrm{~kg}$ : a) beds, b) operating table, c) seats, d) armchairs, e) chairs, f) transport carts, g) weighing machine; 2) visual tracking; 3) devices for electrosurgery - incision and haemostasis during surgery.

Other requirements: In the location of the unit: 1) anaesthesia and intensive care unit; 2) specialist clinic - general surgery; 3 ) radiology facility adapted for examining obese patients.

Anaesthesia equipment - in accordance with the ordinance of the Minister of Health.

In the event of complications/adverse events, the bariatric surgery centre has to ensure that the patient undergoing surgery due to obesity is guaranteed the constant availability ( $24 \mathrm{~h} / 7$ days) to the following:

a) emergency surgical intervention,

b) emergency radiological diagnostics, including computed tomography (CT),

c) emergency laboratory diagnostics

d) intensive care unit (ICU),

e) endoscopy unit ensuring the possibility of emergency diagnostic and therapeutic endoscopy.

All surgeries due to obesity are performed a prio$r i$ by laparoscopic method.

1) At the time of discharge from the centre, the patient has to obtain information about the following:

a) the possibility of permanent, 24-hour contact with a representative of the bariatric team in the postoperative period,

b) dietary recommendations for the period until the next specialist follow-up visit. 


\section{Care standards in the later postoperative period (after 30 days)}

1. The centre performing bariatric procedures has to provide its patients with long-term (unlimited) specialist postoperative care.

2. At the time of discharge from the centre, the patient has to receive a post-operative care plan for a period of 1 year, containing the following dates:

a) at least two surgical follow-up visits (including one up to 30 days after surgery),

b) at least one dietary consultation.

3. During this period, the centre performing procedures in the field of bariatric/metabolic surgery has to ensure the possibility of consultation by a psychologist experienced in the management of bariatric patients.

\section{Conflict of interest}

The authors declare no conflict of interest.

\section{References}

1. Budzyński A, Major P, Głuszek S, et al. Polish recommendations in the field of bariatric and metabolic surgery. Available at: https://www.mp.pl/chirurgia/wytyczne-przegladowe/154894 polskie-rekomendacje-w-zakresie-chirurgii-bariatryczneji-metabolicznej

Received: 3.07.2020, accepted: 4.07.2020. 